\title{
Scaling Law for a Low-Pressure Gas Breakdown in a Homogeneous DC Electric Field
}

\author{
V. A. Lisovsky* and S. D. Yakovin** \\ *Kharkov State University, pl. Svobody 4, Kharkov, 310077 Ukraine \\ **Physics and Technology Center, National Academy of Sciences and Ministry of Education of Ukraine, \\ Kharkov, 310145 Ukraine \\ e-mail: lisovskiy@ftf.univer.kipt.kharkov.ua \\ Received March 15, 2000; in final form, June 5, 2000
}

\begin{abstract}
Gas breakdown in nitrogen, air, and oxygen in a dc electric field at various interelectrode distances $L$ is studied experimentally. A scaling law for a low-pressure gas breakdown $U_{d c}=f(p L, L / R)$ is deduced. According to this scaling law, the breakdown voltage $U_{d c}$ is a function not only of the product of the gas pressure $p$ and the gap length $L$, but also of the ratio of the gap length $L$ to the chamber radius $R$. It is shown that, for any dimensions of the cylindrical discharge chamber (in the range of $L / R$ under investigation), the ratio of the breakdown electric field to the gas pressure $p$ at the minimum of the ignition curve remains constant: $\left(E_{d c} / p\right)_{\min } \approx$ const. A method for calculating the ignition curve in a cylindrical discharge chamber with arbitrary values of $L$ and $R$ is proposed. (C) 2000 MAIK "Nauka/Interperiodica”.
\end{abstract}

PACS numbers: 51.50.+v; 52.80.Hc

As is known [1-7], the ignition curves of a glow discharge are described by the Paschen law $U_{d c}=f(p L)$; i.e., the breakdown voltage $U_{d c}$ is a function of the product of the gas pressure $p$ and the interelectrode distance $L$. The Paschen law implies that the ignition curves $U_{d c}(p)$ measured for various distances $L$ must coincide if they are drawn as the function $U_{d c}(p L)$. However, the measurements of the ignition curves of a glow discharge in neon [8] showed that, with equal values of the product $p L$, the breakdown voltage for a long discharge gap with planar electrodes is significantly higher than that for a short gap. More recent studies [9-14] confirmed this conclusion for some other gases (neon, argon, nitrogen, hydrogen, etc.). In spite of a great number of experimental and theoretical papers devoted to low-pressure gas breakdown in a dc electric field, a method for calculating the ignition curve at arbitrary values of the interelectrode distance $L$ and the radius of the discharge chamber $R$ is still lacking.

This paper is devoted to the experimental study of a breakdown in nitrogen, air, and oxygen in a dc electric field in a discharge chamber with a variable interelectrode distance $L$. It is shown that, in the range of the ratio $L / R$ under study, the ignition curves shift toward high values of the product $p L$ and discharge voltage $U_{d c}$ as the gap length $L$ increases. In this case, for any values of the gap length $L$, the ratio of the breakdown electric field to the gas pressure $\left(E_{d c} / p\right)_{\min }$ at the minimum of the ignition curve remains constant. A generalized scaling law for the low-pressure gas breakdown $U_{d c}=$ $f(p L, L / R)$ is deduced. A method allowing one to calculate the ignition curve for a glow discharge in a cylin- drical chamber with arbitrary dimensions from the known ignition curve for a narrow discharge gap (for $L / R \longrightarrow 0$ ), i.e., from the usual Paschen curve, is described.

We measured the ignition curves for a glow discharge in the range of dc voltages $U_{d c} \leq 1000 \mathrm{~V}$ and pressures of $p \approx 10^{-2}-10$ torr. A discharge tube with an inner diameter of $63 \mathrm{~mm}$ was used. The interelectrode distance $L$ was varied in the range $0.5-10 \mathrm{~cm}$; consequently, the studies were conducted in the range $L / R=$ 0.16-3.2. Planar parallel electrodes spanned the entire cross section of the discharge tube. Both the anode and the cathode were made from stainless steel. The breakdown voltage was measured accurate to $\pm 2 \mathrm{~V}$. When determining the ignition voltage, the growth rate of the discharge voltage did not exceed $1 \mathrm{~V} / \mathrm{s}$. In all cases, our procedure for measuring the ignition curves was as follows. We fixed a certain distance $L$ between the electrodes and then, for various gas pressures $p$, measured the breakdown voltage $U_{d c}$. Below, we explain why only this way of measuring the ignition curves of a glow discharge is correct.

Figure 1 shows the ignition curves measured by us in nitrogen for different distances $L$ between the electrodes. It is seen from the figure that, as $L$ increases, the ignition curves shift not only toward higher ignition voltages $U_{d c}$ (as was obtained in [9-14]), but also toward higher values of $p L$. Apparently, such a shift of the ignition curves toward higher values of $U_{d c}$ and $p L$ with increasing interelectrode distance $L$ may be attributed to an increase in losses of charged particles on the 

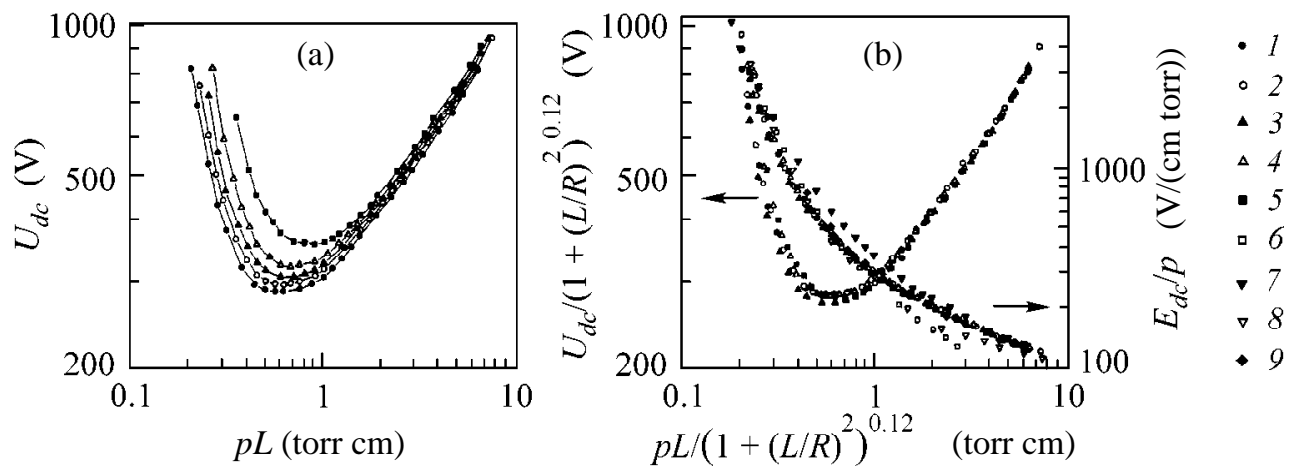

Fig. 1. (a) Experimental ignition curves of a glow discharge in nitrogen for the interelectrode distances $L=(1) 0.5$, (2) 2 , (3) 4 , (4) 6 , and (5) $8 \mathrm{~cm}$. (b) Dependences of $U_{d c}^{*}$ and $E_{d c} / p$ on $(p L)^{*}$ obtained from curves (1)-(5) in Fig. 1a and the data from (6) [11], (7) [15], (8) [16], and (9) [18].

cylindrical wall of the discharge tube due to diffusion across the electric field.

Figure 2 shows the dependences of $U_{\min }$ and $\left(E_{d c} / p\right)_{\min }$ on the value of $(p L)_{\min }$ obtained from our experimental ignition curves measured in a nitrogen discharge by varying the distance $L$. The solid line $U_{\min }=407(p L)_{\min }$ and the dashed line $\left(E_{d c} / p\right)_{\min }=407 \pm$ $5 \mathrm{~V} /(\mathrm{cm}$ torr $)$ describe the results obtained fairly well. Hence, for any distance $L$ between the electrodes, the ratio $\left(E_{d c} / p\right)_{\min }$ at the minimum of the ignition curve remains constant. This is also true if we change the value of the ion-electron emission coefficient $\gamma$ of the cathode material (as was noted in [2,3]).

Note that, by properly choosing the reference axes, we can make all of the obtained ignition curves almost coincide. For example, if we take

$$
\begin{gathered}
(p L)^{*}=p L /\left(1+(L / R)^{2}\right)^{a}, \\
U_{d c}^{*}=U_{d c} /\left(1+(L / R)^{2}\right)^{a},
\end{gathered}
$$

as the abscissa and ordinate, respectively, where $a \approx 0.12$ for nitrogen, then the ignition curves presented in Fig. 1a coincide accurate to $\pm 5 \mathrm{~V}$ (Fig. 1b). It is evident that, for $L / R \longrightarrow 0$, we have the usual Paschen curve $U_{d c}=f(p L)$. It follows from Eqs. (1) and (2) that $U_{d c}^{*} /(p L)^{*}=U_{d c} / p L=E_{d c} / p$; i.e., the dependences $E_{d c} / p=f\left((p L)^{*}\right)$ for different ignition curves must also coincide (which is seen in Fig. 1b). Here, we also see a reasonable agreement between our results and data from [11, 15-18]. For air, we have $a \approx 0.09$ (Fig. 3) and for hydrogen, we have $a \approx 0.03$ (Fig. 4). Note that, in Figs. 3 and 4 , the dependences $E_{d c} / p=f\left((p L)^{*}\right)$ obtained from experimental results $[2,9,15,16,19]$ agree satisfactorily with our data. From our results, it follows that the scaling law for the gas breakdown can be written in the form $\left.U_{d c}=f(p L, L / R)\right)$ or $U_{d c}^{*}=$ $f\left((p L)^{*}\right)$.
Based on Eqs. (1) and (2) and the values of breakdown voltage given in the figures, we can calculate, to a high accuracy, the ignition curves for any cylindrical discharge chamber for arbitrary values of the distance

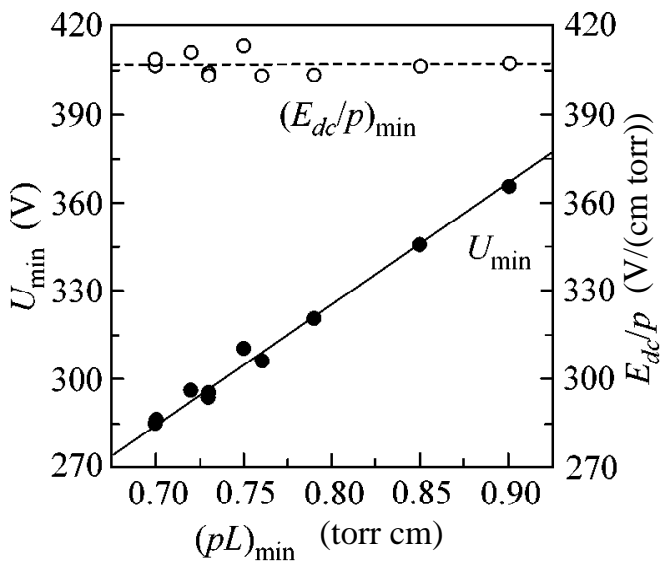

Fig. 2. Dependences of $U_{\min }$ and $\left(E_{d c} / p\right)_{\min }$ on $(p L)_{\min }$ for nitrogen. The solid line corresponds to $U_{\min }=407(p L)_{\min }$, and the dashed line corresponds to $\left(E_{d c} / p\right)_{\min }=407 \mathrm{~V} /(\mathrm{cm}$ torr).

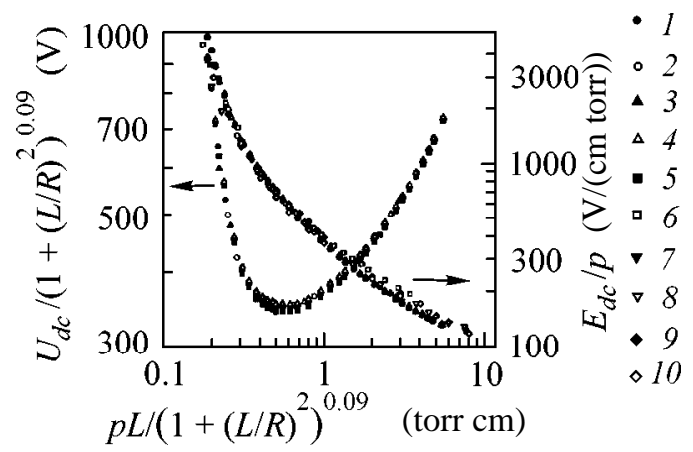

Fig. 3. Dependences of $U_{d c}^{*}$ and $E_{d c} / p$ on $(p L) *$ for a glow discharge in air for the interelectrode distances $L=(1) 0.5$, (2) $1,(3) 2$, (4) 5 , and (5) $10 \mathrm{~cm}$ and the data from (6) [15], (7) [19], (8) [16], and (9) [20], and (10) [21]. 


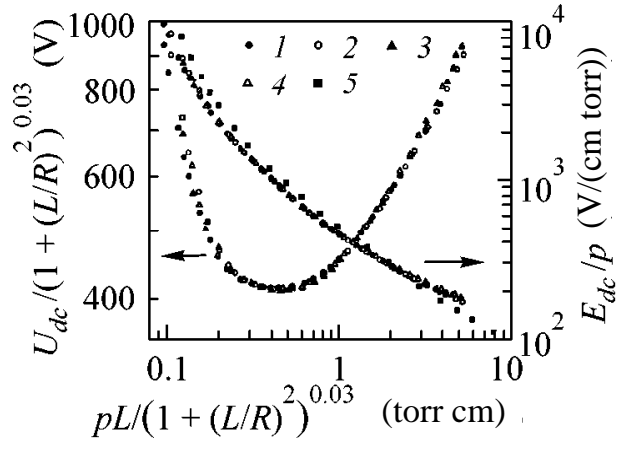

Fig. 4. Dependences of $U_{d c}^{*}$ and $E_{d c} / p$ on $(p L)^{*}$ for a glow discharge in oxygen for the interelectrode distances $L=(1) 0.5$, (2) 3, (3) 6, (4) $8 \mathrm{~cm}$ and (5) the data from [15].

$L$ and radius $R$. As an example, we find the position of the minimum in the ignition curve for a nitrogen discharge for $R=3.15 \mathrm{~cm}, L=5 \mathrm{~cm}$, and a stainless-steel cathode. From Fig. $1 \mathrm{~b}$, it is seen that $U_{\min }^{*} \approx 280 \mathrm{~V}$ and $(p L)_{\min }^{*} \approx 0.6$ torr $\mathrm{cm}$. From (1) and (2), we obtain

$$
\begin{aligned}
(p L)_{\min } & =(p L)_{\min }^{*}\left[1+(L / R)^{2}\right]^{0.12}, \\
U_{\min } & =U_{\min }^{*}\left[1+(L / R)^{2}\right]^{0.12} .
\end{aligned}
$$

For $L / R=1.59$, we have $U_{\min } \approx 323 \mathrm{~V}$ and $(p L)_{\min } \approx$ 0.7 torr $\mathrm{cm}$. From our experimental results, it follows that $U_{\min } \approx 320 \mathrm{~V}$ and $(p L)_{\min } \approx 0.73$ torr $\mathrm{cm}$; i.e., there is a good agreement between the coordinates of the maximum in the ignition curve obtained experimentally and those calculated using Eqs. (1)-(4). To calculate any other point on the ignition curve $U_{1}^{*}$ and $(p L)_{1}^{*}$, we use the same procedure. In the general case, to calculate the ignition curve in a cylindrical discharge chamber with arbitrary dimensions, one should have the Paschen curve (i.e., the ignition curve measured in a discharge chamber such that $L / R \longrightarrow 0$ and with the same cathode material). Then, from Eqs. (1) and (2), one can determine the values of $U_{d c}$ and $p L$. If the initial ignition curve has been measured in a discharge chamber with $L_{0}$ and $R_{0}$, such that the condition $L_{0} / R_{0} \longrightarrow 0$ does not hold, one should first calculate the dependence $U_{d c}^{*}=f\left((p L)^{*}\right)$ using Eqs. (1) and (2) and then, from the same relations, calculate the ignition curve for a discharge chamber with the given dimensions $L_{1}$ and $R_{1}$. This may be written more concisely in the following form:

$$
(p L)_{1}=p L_{0}\left[\frac{1+\left(L_{1} / R_{1}\right)^{2}}{1+\left(L_{0} / R_{0}\right)^{2}}\right]^{a},
$$

$$
U_{d c 1}=U_{d c 0}\left[\frac{1+\left(L_{1} / R_{1}\right)^{2}}{1+\left(L_{0} / R_{0}\right)^{2}}\right]^{a},
$$

where the index " 1 " stands for the ignition curve to be sought and the index " 0 " stands for the initially known ignition curve.

The ignition curve of a glow discharge is usually measured by two methods: (i) the distance $L$ is fixed, and the breakdown voltage $U_{d c}$ is measured at different values of the gas pressure $p$; and (ii) the value of $p$ is fixed, and the breakdown voltage $U_{d c}$ is measured at different values of the distance $L$. However, the results obtained in this study show that the latter method of measuring the ignition curve (at a fixed value of $p$ and variable $L$ ) is incorrect. The ignition curves obtained in this way are close to the Paschen curve only at small values of $L$ but shift toward higher breakdown voltages with increasing $L$. Hence, the ignition curve of a glow discharge must be measured by varying the gas pressure $p$, the distance $L$ between the electrodes being fixed.

In summary, the ignition of a glow discharge in nitrogen, air, and oxygen at a variable interelectrode distance $L$ have been studied experimentally. It is shown that, at any interelectrode distance $L$, the ratio $\left(E_{d c} / p\right)_{\min }$ at the minimum of the ignition curve remains constant. In the range of $L / R$ under study, the scaling law for gas breakdown $U_{d c}=f(p L, L / R)$ holds; i.e., the breakdown voltage $U_{d c}$ is a function not only of the product of the gas pressure $p$ and the gap length $L$, but also of the ratio $L / R$. A method for calculating the ignition curve in a cylindrical discharge chamber with arbitrary values of $L$ and $R$ is proposed.

\section{REFERENCES}

1. F. Paschen, Ann. Phys. Chem., Ser. 3 37, 69 (1889).

2. M. J. Druyvesteyn and F. M. Penning, Rev. Mod. Phys. 12, 87 (1940).

3. J. M. Meek and J. D. Craggs, Electrical Breakdown of Gases (Clarendon, Oxford, 1953; Inostrannaya Literatura, Moscow, 1960).

4. Yu. P. Raŭzer, Gas Discharge Physics (Nauka, Moscow, 1987).

5. V. A. Lisovsky and V. D. Yegorenkov, J. Phys. D 27, 2340 (1994).

6. A. V. Phelps and Z. Lj. Petrovic, Plasma Sources Sci. Technol. 8, R21 (1999).

7. M. Sato, Bull. Yamagata Univ. 25, 119 (1999).

8. J. S. Townsend and S. P. McCallum, Philos. Mag. 6, 857 (1928).

9. H. Fricke, Z. Phys. 86, 464 (1933).

10. S. P. McCallum and L. Klatzow, Philos. Mag. 17, 279 (1934).

11. H. C. Miller, Physica (Amsterdam) 30, 2059 (1964). 
12. L. Jacques, W. Bruynooghe, R. Boucique, and W. Wieme, J. Phys. D 19, 1731 (1986).

13. M. Yumoto, T. Sakai, Y. Ebinuma, et al., in Proceedings of the 8th International Symposium on High-Voltage Engineering, Yokohama, 1993, p. 409.

14. G. Auday, P. Guillot, J. Galy, and H. Brunet, J. Appl. Phys. 83, 5917 (1998).

15. M. J. Schonhuber, IEEE Trans. Power Appar. Syst. 88, 100 (1969).

16. T. W. Dakin, J. Gerhold, Z. Krasucki, et al., in Proceedings of the International Conference on Large HighVoltage Electric Systems, Paris, 1977, p. 1.
17. B. Held, N. Soulem, R. Peyrous, and N. Spyrou, Trans. Inst. Electr. Eng. Jpn., Part A 116, 925 (1996).

18. B. Held, N. Soulem, R. Peyrous, and N. Spyrou, J. Phys. III 7, 2059 (1997).

19. J. A. Pim, Proc. Inst. Electr. Eng., Part 3 96, 117 (1949).

20. S. C. Brown, in Basic Data of Plasma Physics (Wiley, New York, 1959), p. 240.

21. H. Ritz, Arch. Elektrotech. (Berlin) 26, 219 (1937).

Translated by N. Larionova 